\title{
Connectedness of suborbital graphs for a special subgroup of the modular group
}

\author{
Murat Beşenk
}

(Communicated by Murat TOSUN)

\begin{abstract}
In this paper, we investigate connectedness of suborbital graphs for a special congruence subgroups.Firstly, conditions for being an edge self-paired are provided, then in order to make graph connected, we give necessary and sufficient conditions for the $£_{u, n}$, whose vertices form the block [ $\infty$ ].
\end{abstract}

Keywords: Modular group; Imprimitive action; Suborbital graph; Connectedness.

AMS Subject Classification (2010): Primary: 05C05 ; Secondary: 05C20; 11F06; 20H05; $20 \mathrm{H} 10$.

*Corresponding author

\section{Introduction}

Let $\operatorname{PSL}(2, \mathbb{R})$ denote the group consisting of all linear fractional transformations

$$
\theta: \tau \rightarrow \frac{a \tau+b}{c \tau+d}, \text { where } a, b, c, d \text { are real numbers and } a d-b c=1 .
$$

In terms of matrix representation, the elements of $\operatorname{PSL}(2, \mathbb{R})$ correspond to the matrices

$$
\pm\left(\begin{array}{ll}
a & b \\
c & d
\end{array}\right) ; a, b, c, d \text { are real numbers and } a d-b c=1 .
$$

The modular group $\Gamma=P S L(2, \mathbb{Z})$, is the subgroup of $\operatorname{PSL}(2, \mathbb{R})$ with integral coefficients. It is generated by the matrices

$$
U=\left(\begin{array}{cc}
0 & -1 \\
1 & 0
\end{array}\right) ; \quad V=\left(\begin{array}{cc}
0 & -1 \\
1 & 1
\end{array}\right)
$$

with defining relationships $U^{2}=V^{3}=-I$, where $I$ is the identity matrix. This is the automorphism group of the upper half plane $\mathbb{H}:=\{\tau \in \mathbb{C}: \operatorname{Im}(\tau)>0\} . \Gamma$ will denote the modular group, a special subgroup of $P S L(2, \mathbb{R})$ with integral coefficients. $\Gamma$ is a Fuchsian group whose fundamental domain has finite area, so it has a signature consisting of the geometric invariants $\left(g ; m_{1}, \ldots, m_{r}, s\right)$ where $g$ is the genus of the compactified quotient space,

Received : 11-August-2015, Accepted : 18-Mart-2016

This article is the written version of author's plenary talk delivered on September 03-August 31, 2015 at 4th International Eurasian Conference on Mathematical Sciences and Applications (IECMSA-2015) in Athens, Greece. 
$m_{1}, \ldots, m_{r}$ are the periods of the elliptic elements and $s$ is the parabolic class number. $\Gamma$ has the signature $(0 ; 2,3, \infty)$, so it is isomorphic to a free product $C_{2} * C_{3}$. The signature of a discrete Fuchsian group is a very interesting problem in group theory and arithmetic-algebraic geometry.In a paper [1] Jones, Singerman and Wicks showed that the well-known Farey Graph is an example of a suborbital graph. Then, a number of papers on suborbital graphs for related groups were published. These papers point out the relation between elliptic elements and circuits in graphs. This fact is important because it means that suborbital graphs have a potential to clarify signature problems.

\section{Preliminaries}

The principal congruence subgroup of $\Gamma$ is defined to be the subgroup

$$
\Gamma(n)=\left\{\left(\begin{array}{ll}
a & b \\
c & d
\end{array}\right) \in \Gamma: a \equiv d \equiv 1(\bmod n), b \equiv c \equiv 0(\bmod n)\right\} .
$$

A subgroup of $\Gamma$ is called a congruence group provided it contains the principal congruence group $\Gamma(n)$.Congruence groups have been of great interest in many fields of mathematics, number theory, group theory, etc.

Jones, Singerman and Wicks [1] used the notion of the imprimitive action [3],[4], [9] for a $\Gamma$ - invariant equivalence relation induced on $\mathbb{Q} \cup\{\infty\}$ by the congruence subgroup $\Gamma_{0}(n)=\left\{\left(\begin{array}{ll}a & b \\ c & d\end{array}\right) \in \Gamma: c \equiv 0(\bmod n)\right\}$ to obtain some suborbital graphs and examined their connectedness and forest properties.Some applications of this method can be found in the papers,[1],[2].Particularly in [2],[12],[13] and [14] authors give some results about a connection between the periods of elliptic elements of chosen permutation group with the circuits in suborbital graphs of it. In this article we introduce a different invariant equivalence relation by using the congruence subgroup $\Gamma^{*}(n)=\left\{\left(\begin{array}{ll}a & b \\ c & d\end{array}\right) \in \Gamma: b \equiv c \equiv 0(\bmod n)\right\}$ which is well known in [6], instead of $\Gamma_{0}(n)$ and obtain some results for the newly constructed subgraphs $£_{u, n}$. Our results for $\Gamma^{*}(n)$ may help to confirm above main idea. Also, it is worth noting that these concepts are very much related to the binary quadratic forms and modular forms in [6],[10],[11]. In this study, we consider the action of the group $\Gamma^{*}(n)$ on the set $\hat{\mathbb{Q}}_{n}:=\left\{\frac{a}{c n}:(a, c n)=1 ; a, c \in \mathbb{Z}\right\}$ in the spirit of the theory of permutation groups, and graph arising from this action in hyperbolic geometric terms.

Every element of $\hat{\mathbb{Q}}_{n}$ can be represented as a reduced fraction $\frac{x}{y}$ with $x, y \in \mathbb{Z}$ and $(x, y)=1$. Also $\infty$ is represented as $\frac{1}{0}=\frac{-1}{0}$. The action of $\Gamma^{*}(n)$ on $\hat{\mathbb{Q}}_{n}$ now becomes

$$
\left(\begin{array}{cc}
a & b n \\
c n & d
\end{array}\right):\left(\begin{array}{l}
x \\
y
\end{array}\right) \rightarrow \frac{a x+b n y}{c n x+d y}
$$

Thus the matrix $\left(\begin{array}{cc}a & b n \\ c n & d\end{array}\right)$ on $\frac{x}{y}$ and $\frac{-x}{-y}$ are identical. If the determinant of the matrix $\left(\begin{array}{cc}a & b n \\ c n & d\end{array}\right)$ is 1 and $(x, y)=1$, then $(a x+b y, c x+d y)=1$.

Theorem 2.1. The action of $\Gamma^{*}(n)$ on $\hat{\mathbb{Q}}_{n}$ is transitive.

Proof. It is enough to prove that the orbit containing $\infty$ is $\hat{\mathbb{Q}}_{n}$. If $\frac{a}{b n} \in \hat{\mathbb{Q}}_{n}$ then there exist $\alpha, \beta \in \mathbb{Z}$ such that $a \alpha-b \beta n^{2}=1$. Then the element $\left(\begin{array}{cc}a & \beta n \\ b n & \alpha\end{array}\right)$ is in $\hat{\mathbb{Q}}_{n}$ and sends $\infty$ to $\left(\begin{array}{c}a \\ b n\end{array}\right)$.

Lemma 2.1. The stabilizer of $\infty$ in $\hat{\mathbb{Q}}_{n}$ is the set $\left\{\left(\begin{array}{ll}1 & \lambda \\ 0 & 1\end{array}\right): \lambda \in \mathbb{Z}\right\}$ denoted by $\Gamma^{*}(n)_{\infty}$.

Proof. The stabilizer of a point in $\hat{\mathbb{Q}}_{n}$ is a infinite cyclic group. Since the action is transitive, stabilizers of any two points are conjugate. Therefore it is enough to look at the stabilizer of $\infty$ in $\Gamma^{*}(n)$. Let $T \in \Gamma^{*}(n)$ then

$$
T\left(\begin{array}{l}
1 \\
0
\end{array}\right)=\left(\begin{array}{cc}
a & b n \\
c n & d
\end{array}\right)\left(\begin{array}{l}
1 \\
0
\end{array}\right)=\left(\begin{array}{l}
1 \\
0
\end{array}\right)
$$


and so $\left(\begin{array}{c}a \\ c n\end{array}\right)=\left(\begin{array}{l}1 \\ 0\end{array}\right)$, then $a=d=1, c=0$ and $b n=\lambda \in \mathbb{Z}$. Hence $T=\left(\begin{array}{ll}1 & \lambda \\ 0 & 1\end{array}\right)$. This shows that the stabilizer of $\infty$ in $\Gamma^{*}(n)$ is

$$
\Gamma^{*}(n)_{\infty}:=\left\{\left(\begin{array}{cc}
1 & b n \\
0 & 1
\end{array}\right): n \in \mathbb{Z}\right\}=\left\langle\left(\begin{array}{cc}
1 & n \\
0 & 1
\end{array}\right)\right\rangle .
$$

We now consider the imprimitivity of the action of $\Gamma^{*}(n)$ on $\hat{\mathbb{Q}}_{n}$, beginning with a general discussion of primitivity of permutation groups. Let $(G, \Omega)$ be a transitive permutation group, consisting of a group $G$ acting on a set $\Omega$ transitively. An equivalence relation $\approx$ on $\Omega$ is called $G$-invariant if, whenever $\alpha, \beta \in \Omega$ satisfy $\alpha \approx \beta$, then $h(\alpha) \approx h(\beta)$ for all $h \in G$. The equivalence classes are called blocks, and the block containing $\alpha$ is denoted by $[\alpha]$.

We call $(G, \Omega)$ imprimitive if $\Omega$ admits some $G$-invariant equivalence relation different from

(i) the identity relation, $\alpha \approx \beta$ if and only if $\alpha=\beta$;

(ii) the universal relation, $\alpha \approx \beta$ for all $\alpha, \beta \in \Omega$.

Otherwise $(G, \Omega)$ is called primitive. These two relations are supposed to be trivial relations. Clearly, a primitive group must be transitive, if not, the orbits would form a system of blocks. The converse is false, but we have the following useful result in [3].

Lemma 2.2. Let $(G, \Omega)$ be a transitive permutation group. $(G, \Omega)$ is primitive if and only if $G_{\alpha}$, the stabilizer of $\alpha \in \Omega$, is a maximal subgroup of $G$ for each $\alpha \in \Omega$.

From the above lemma we see that whenever, for some $\alpha, G_{\alpha}<H<G$, then $\Omega$ admits some $G$-invariant equivalence relation other than the trivial cases. Because of the transitivity, every element of $\Omega$ has the form $h(\alpha)$ for some $h \in G$. Thus one of the non-trivial $G$-invariant equivalence relation on $\Omega$ by $H$ is given as follows:

$$
h_{1}(\alpha) \approx h_{2}(\alpha) \text { if and only if } h_{2} \in h_{1} H .
$$

The number of blocks (equivalence classes ) is the index $|G: H|$ and the block containing $\alpha$ is just the orbit $H(\alpha)$.

We can apply these ideas to the case where $G$ is the $\Gamma^{*}(n)$ and $\Omega$ is $\hat{\mathbb{Q}}_{n}$. It is clear that $\Gamma^{*}(n)_{\infty}<\Gamma(n)<\Gamma^{*}(n)$. We shall define an equivalence relation $\approx$ induced on $\hat{\mathbb{Q}}_{n}$ by $\Gamma^{*}(n)$ for $n \in \mathbb{N}$ and $n>1$. Now let $\frac{r}{s n}, \frac{x}{y n} \in \hat{\mathbb{Q}}_{n}$. Corresponding to these there are two matrices

$$
T_{1}:=\left(\begin{array}{cc}
r & r_{0} n \\
s n & s_{0}
\end{array}\right), \quad T_{2}:=\left(\begin{array}{cc}
x & x_{0} n \\
y n & y_{0}
\end{array}\right)
$$

in $\Gamma^{*}(n)$ for which $T_{1}(\infty)=\frac{r}{s n}$ and $T_{2}(\infty)=\frac{x}{y n}$. We get the following imprimitive $\Gamma^{*}(n)$ - invariant equivalence relation on $\hat{\mathbb{Q}}_{n}$ by $\Gamma(n)$ as

$$
T_{1}(\infty) \approx T_{2}(\infty) \quad \text { if and only if } T_{1}^{-1} T_{2} \in \Gamma(n),
$$

and so from the above we can easily verify that $\frac{r}{s n} \approx \frac{x}{y n}$ if and only if $x \equiv r(\bmod n)$. Indeed, let $\frac{r}{s n} \approx \frac{x}{y n}$. Then $T_{1}(\infty) \approx T_{2}(\infty)$ and

$$
T_{1}^{-1} T_{2}=\left(\begin{array}{cc}
s_{0} & -r_{0} n \\
-s n & r
\end{array}\right)\left(\begin{array}{cc}
x & x_{0} n \\
y n & y_{0}
\end{array}\right)=\left(\begin{array}{cc}
x s_{0}-y r_{0} n^{2} & s_{0} x_{0} n-r_{0} y_{0} n \\
-s n x+r y n & -s x_{0} n^{2}+r y_{0}
\end{array}\right) \in \Gamma(n) .
$$

Hence these equations $x s_{0}-y r_{0} n^{2} \equiv-s x_{0} n^{2}+r y_{0} \equiv 1(\bmod n)$ and $-s n x+r y n \equiv s_{0} x_{0} n-r_{0} y_{0} n \equiv 0(\bmod n)$ are obtained. So $x s_{0} \equiv r y_{0} \equiv 1(\bmod n)$. Also, since $T_{1}, T_{2} \in \Gamma^{*}(n) \subset \Gamma, r s_{0}-s r_{0} n^{2}=1$ and $x y_{0}-y x_{0} n^{2}=1$. Thus $r s_{0} \equiv 1(\bmod n)$ and $x y_{0} \equiv 1(\bmod n)$. Since $x s_{0} \equiv 1(\bmod n)$ then $x r s_{0} \equiv r(\bmod n)$ and also $x \equiv r(\bmod n)$ is achieved. Similarly if we use the equation $r y_{0} \equiv 1(\bmod n)$, then we get the same result.

Conversely, let $x \equiv r(\bmod n)$. Since $\frac{r}{s n} \in \hat{\mathbb{Q}}_{n},(r, s n)=1$ and $\left(r, s n^{2}\right)=1$. Then there exist $r_{0}, s_{0} \in \mathbb{Z}$ such that $r s_{0}-s n^{2} r_{0}=1$. Similarly, for $\frac{x}{y n} \in \hat{\mathbb{Q}}_{n}, x y_{0}-y n^{2} x_{0}=1$ is satisfied. Thus $T_{1}:=\left(\begin{array}{cc}r & r_{0} n \\ s n & s_{0}\end{array}\right), T_{2}:=$ $\left(\begin{array}{cc}x & x_{0} n \\ y n & y_{0}\end{array}\right) \in \Gamma^{*}(n)$. Moreover from the above equations we have $r s_{0} \equiv 1(\bmod n)$ and $x y_{0} \equiv 1(\bmod n)$. Also, 
since $x \equiv r(\bmod n)$ then $r y_{0} \equiv 1(\bmod n)$ and so $r y_{0}-s x_{0} n^{2} \equiv 1(\bmod n)$. Again, likewise $x s_{0}-y r_{0} n^{2} \equiv 1(\operatorname{modn})$ is obtained. Consequently, these cases show that $\left(\begin{array}{cc}r & r_{0} n \\ s n & s_{0}\end{array}\right)^{-1}\left(\begin{array}{cc}x & x_{0} n \\ y n & y_{0}\end{array}\right) \in \Gamma(n)$. Then $T_{1}(\infty) \approx T_{2}(\infty)$ and so $\frac{r}{s n} \approx \frac{x}{y n}$. The number $\psi(n)$ of equivalence class under $\approx$ is given by $\psi(n)=\left|\Gamma^{*}(n): \Gamma(n)\right|$. So the block of $\infty$ is obtained as

$$
[\infty]:=\left\{\frac{x}{y n} \in \hat{\mathbb{Q}}_{n} \mid x \equiv 1(\bmod n)\right\} .
$$

\section{Suborbital Graphs of $\Gamma^{*}(n)$ on $\hat{\mathbb{Q}}_{n}$}

In [4], Sims introduced the idea of the suborbital graphs of a permutation group $G$ acting on a set $\Omega$, these are graphs with vertex-set $\Omega$, on which $G$ induces automorphisms. We summarise Sims' theory as follows:

Let $(G, \Omega)$ be transitive permutation group. Then $G$ acts on $\Omega \times \Omega$ by

$$
\Theta: \underset{(g,(\alpha, \beta))}{\Theta \times(\Omega \times \Omega)} \rightarrow \underset{(g(\alpha), g(\beta))}{\Omega}
$$

where $g \in G$ and $\alpha, \beta \in \Omega$. The orbits of this action are called suborbitals of $G$. The orbit containing $(\alpha, \beta)$ is denoted by $O(\alpha, \beta)$. From $O(\alpha, \beta)$ we can form a suborbital graph $G(\alpha, \beta)$ : its vertices are the elements of $\Omega$, and there is a directed edge from $\gamma$ to $\delta$ if $(\gamma, \delta) \in O(\alpha, \beta)$. A directed edge from $\gamma$ to $\delta$ is denoted by $\gamma \rightarrow \delta$. If $(\gamma, \delta) \in O(\alpha, \beta)$, then we will say that there exists an edge $\gamma \rightarrow \delta$ in $G(\alpha, \beta)$. This theory reveals the relationship between graphs and permutation groups. In this paper our calculation concerns $\Gamma^{*}(n)$, so we can draw this edge as a hyperbolic geodesic in the upper half-plane $\mathbb{H}$, that is, as Euclidean semi-circles and half-lines perpendicular to the real line.

The orbit $O(\beta, \alpha)$ is also a suborbital graph and it is either equal to or disjoint from $O(\alpha, \beta)$. In the latter case $G(\beta, \alpha)$ is just $G(\alpha, \beta)$ with the arrows reserved and we call, in this case, $G(\alpha, \beta)$ and $G(\beta, \alpha)$ paired suborbital graphs. In the former case $G(\alpha, \beta)=G(\beta, \alpha)$ and the graph consists of pairs of oppositely directed edges; it is convenient to replace each such pair by a single undirected edge, so that we have an undirected graph which we call self paired.

Definition 3.1. By a directed circuit in $G_{u, m n}$ we mean that a sequence $v_{1}, v_{2}, \ldots, v_{k}$ of different vertices such that $v_{1} \longrightarrow v_{2} \longrightarrow \ldots \longrightarrow v_{k} \longrightarrow v_{1}$, where $k \in \mathbb{N}$ and $k \geq 3$; an anti-directed circuit will denote a configuration like the above with at least an arrow ( not all ) reversed.

If $m=2$, then we will say the configuration $v_{1} \longrightarrow v_{2} \longrightarrow v_{1}$ a self paired edge: it consists of a loop based at each vertex.

If $m=3$ or $m=4$, then the circuit, directed or not, is called a triangle or quadrilateral.

A graph which contains no circuit is called a forest.

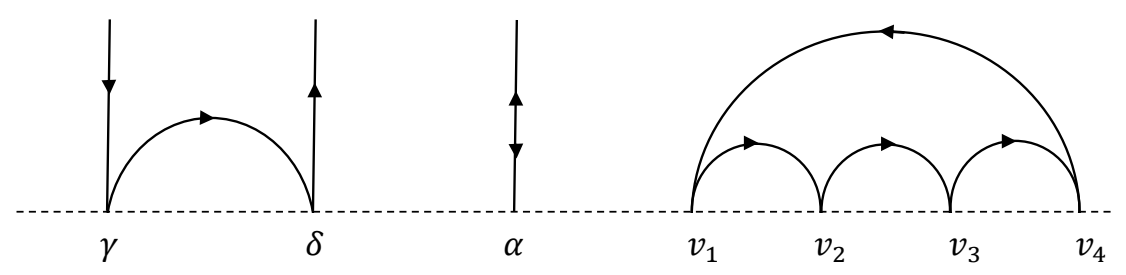

Figure 1. Circuits

Since $\Gamma^{*}(n)$ acts transitively on $\hat{\mathbb{Q}}_{n}$, each suborbital contains a pair $(\infty, v)$ for some $v \in \hat{\mathbb{Q}}_{n}$; writing $v=\frac{u}{m n}$ with $(u, m n)=1$ we denote this suborbital by $O_{u, m n}$ and the corresponding suborbital graph by $G_{u, m n}$. If $v=\infty$, we would have the simplest suborbital graph, namely $G_{1,0}=G_{-1,0}$. The above ideas are also described in [8].

\section{Graph $\mathrm{G}_{u, n}$}

We now investigate the suborbital graphs for the action $\Gamma^{*}(n)$ on $\hat{\mathbb{Q}}_{n}$. 
Lemma 3.1. Let $v, v^{\prime} \in \hat{\mathbb{Q}}_{n} . O(\infty, v)=O\left(\infty, v^{\prime}\right)$ if and only if $v, v^{\prime}$ in the same orbit of $\Gamma^{*}(n)_{\infty}$. That is, there exists $g \in \Gamma^{*}(n)$ such that $g(v)=v^{\prime}$.

Proof. Assume that $O(\infty, v)=O\left(\infty, v^{\prime}\right)$. Then $(\infty, v) \in O\left(\infty, v^{\prime}\right)$.Therefore there exists $g \in \Gamma^{*}(n)$ such that $g(\infty, v)=\left(\infty, v^{\prime}\right)$. Since $(g(\infty), g(v))=g(\infty, v)=\left(\infty, v^{\prime}\right)$ then $(g(\infty), g(v))=\left(\infty, v^{\prime}\right)$ and $g(\infty)=\infty, g(v)=v^{\prime}$.So $g \in \Gamma^{*}(n)_{\infty}$.

Conversely, we suppose that $v, v^{\prime}$ in the same orbit of $\Gamma^{*}(n)_{\infty}$. Then there exists $g \in \Gamma^{*}(n)_{\infty}$ such that $g(\infty)=$ $\infty, g(v)=v^{\prime}$.Hence $g(\infty, v)=(g(\infty), g(v))=\left(\infty, v^{\prime}\right) \in O\left(\infty, v^{\prime}\right)$. Thus $(\infty, v) \in O\left(\infty, v^{\prime}\right)$ and $O(\infty, v) \cap O\left(\infty, v^{\prime}\right) \neq$ $\emptyset$ are obtained.In this way $O(\infty, v)=O\left(\infty, v^{\prime}\right)$ is achieved.

Corollary 3.1. $G_{u, m n}=G_{u^{\prime}, m^{\prime} n}$ if and only if $m=m^{\prime}$ and $u^{\prime} \equiv u\left(\bmod m n^{2}\right)$.

Proof.

Suppose that $O(\infty, v)=O\left(\infty, v^{\prime}\right)$. Then $(\infty, v) \in O\left(\infty, v^{\prime}\right)$. Let $v=\frac{u}{m n}, v^{\prime}=\frac{u^{\prime}}{m^{\prime} n}$. So there exists $g \in \Gamma^{*}(n)_{\infty}$ such that $g(\infty)=\infty$ and $g\left(\frac{u^{\prime}}{m^{\prime} n}\right)=\frac{u}{m n}$. Since $\Gamma^{*}(n)_{\infty}$ is generated by $z: v \rightarrow v+n$, there exists $k \in \mathbb{Z}$ such that $\frac{u}{m n}=g\left(\frac{u^{\prime}}{m^{\prime} n}\right)=\frac{u^{\prime}}{m^{\prime} n}+k n$.Thus

$$
v=\frac{u}{m n}=g\left(\frac{u^{\prime}}{m^{\prime} n}\right)=\frac{u^{\prime}}{m^{\prime} n}+k n=\frac{u^{\prime}+k m n^{2}}{m^{\prime} n} \Leftrightarrow m=m^{\prime}
$$

and

$$
u^{\prime}+k m n^{2}=u \Leftrightarrow m=m^{\prime}, u^{\prime} \equiv u\left(\bmod m n^{2}\right) .
$$

Corollary 3.2. For each integer $n \geq 1$ there are $\psi\left(m n^{2}\right)$ distinct suborbital graphs $G_{u, m n}$ one for some unit $u \in U_{m n^{2}}$.

We will write $\frac{r}{s n} \rightarrow \frac{x}{y n}$ in $G_{u, m n}$ if $\left(\frac{r}{s n}, \frac{x}{y n}\right) \in O_{u, m n}$, that is, if there is a directed edge from $\frac{r}{s n}$ to $\frac{x}{y n}$ in $G_{u, m n}$. We use the following theorem frequently in our calculation.

Theorem 3.1. $\frac{r}{s n} \rightarrow \frac{x}{y n}$ in $G_{u, m n}$ if and only if either

(i) $x \equiv u r\left(\operatorname{modm} n^{2}\right), \quad y \equiv u s(\operatorname{modm})$ and $r y-s x=m$ or

(ii) $x \equiv-u r\left(\bmod m n^{2}\right), \quad y \equiv-u s(\bmod m)$ and $r y-s x=-m$.

Proof. If $\frac{r}{s n} \rightarrow \frac{x}{y n}$ in $G_{u, m n}$, then there exists some $g \in \Gamma^{*}(n)$ such that $g$ sends the pair $\left(\frac{1}{0}, \frac{u}{m n}\right)$ to the pair $\left(\frac{r}{s n}, \frac{x}{y n}\right)$; that is, $g(\infty)=\frac{r}{s n}=\frac{-r}{-s n}$ and $g\left(\frac{u}{m n}\right)=\frac{x}{y n}=\frac{-x}{-y n}$ for $g=\left(\begin{array}{cc}a & b n \\ c n & d\end{array}\right) \in \Gamma^{*}(n)$, $\operatorname{det} g=1$. So we have the matrix equation

$$
\pm\left(\begin{array}{cc}
a & b n \\
c n & d
\end{array}\right)\left(\begin{array}{cc}
1 & u \\
0 & m n
\end{array}\right)=\left(\begin{array}{cc}
r & x \\
s n & y n
\end{array}\right)
$$

If the plus sign is valid, then $a=r, c=s, x=a u+b m n^{2}$ and $y=c u+d m$, so that $x \equiv u r\left(\operatorname{modmn^{2}}\right), y \equiv$ $u s(\bmod m)$.Taking determinants in the matrix equation we see that $r y-s x=m$. Similarly, if the minus sign is valid, we satisfy $(i)$.

Conversely, let be $x \equiv u r\left(\bmod m n^{2}\right), \quad y \equiv u s(\bmod m)$ and $r y-s x=m$. We get $g:=\left(\begin{array}{cc}r & b n \\ s n & d\end{array}\right)$. Then $g(\infty)=$ $\left(\begin{array}{cc}r & b n \\ s n & d\end{array}\right)\left(\begin{array}{l}1 \\ 0\end{array}\right)=\left(\begin{array}{c}r \\ s n\end{array}\right)$ and $g\left(\begin{array}{c}u \\ m n\end{array}\right)=\left(\begin{array}{cc}r & b n \\ s n & d\end{array}\right)\left(\begin{array}{c}u \\ m n\end{array}\right)=\left(\begin{array}{c}x \\ y n\end{array}\right)$. So $g\left(\infty, \frac{u}{m n}\right)=\left(\frac{r}{s n}, \frac{x}{y n}\right)$ is achieved.Then there exist $b, d \in \mathbb{Z}$ such that $x=u r+b m n^{2}, y=u s+d m$. If we put these equivalence in $r y-s x=m$, we obtain $r(u s+d m)-s\left(u r+b m n^{2}\right)=m$. Since

$$
\left(\begin{array}{cc}
r & b n \\
s n & d
\end{array}\right)\left(\begin{array}{cc}
1 & u \\
0 & m n
\end{array}\right)=\left(\begin{array}{cc}
r & u r+b m n^{2} \\
s n & u s n+d m n
\end{array}\right),
$$

we have $r d-b s n^{2}=1$. Consequently, $g \in \Gamma^{*}(n)$.

We can prove case (ii) similarly. 
Theorem 3.2. $G_{u, m n}$ is self-paired if and only if $u^{2}+1 \equiv 0\left(\bmod m n^{2}\right)$.

Proof. We suppose that $G_{u, m n}$ is self-paired.Then

$$
O\left(\infty, \frac{u}{m n}\right)=O\left(\frac{u}{m n}, \infty\right) .
$$

Therefore there are $T \in \Gamma^{*}(n)$ such that $T\left(\infty, \frac{u}{m n}\right)=\left(\frac{u}{m n}, \infty\right)$. So

$$
\left(\begin{array}{cc}
a & b n \\
c n & d
\end{array}\right)\left(\begin{array}{cc}
1 & u \\
0 & m n
\end{array}\right)=\left(\begin{array}{cc}
u & 1 \\
m n & 0
\end{array}\right) .
$$

Thus $a=u, c=m, a u+b m n^{2}=1$ and $c n u+d m n=0$. If $c=m$ then $m n(u+d)=0$. Since $m n>0, d=-u$ is held. So

$$
T=\left(\begin{array}{cc}
a & b n \\
c n & d
\end{array}\right)=\left(\begin{array}{cc}
u & b n \\
m n & -u
\end{array}\right)
$$

and $\operatorname{det} T=-u^{2}-b m n^{2}=1$. As a result we have that $u^{2}+1 \equiv 0\left(m o d m n^{2}\right)$.

Conversely, assume that $u^{2}+1 \equiv 0\left(\bmod m n^{2}\right)$. There exists some integer $b$ such that $-u^{2}-b m n^{2}=1$. Hence $S:=\left(\begin{array}{cc}u & b n \\ m n & -u\end{array}\right)$. So $\left(\begin{array}{cc}u & b n \\ m n & -u\end{array}\right)\left(\begin{array}{l}1 \\ 0\end{array}\right)=\left(\begin{array}{c}u \\ m n\end{array}\right)$, and $\left(\begin{array}{cc}u & b n \\ m n & -u\end{array}\right)\left(\begin{array}{c}u \\ m n\end{array}\right)=\left(\begin{array}{c}-1 \\ 0\end{array}\right)=\left(\begin{array}{l}1 \\ 0\end{array}\right)$ are obtained.That is, $S(\infty)=\frac{u}{m n}$ and $S\left(\frac{u}{m n}\right)=\infty$. Consequently $O\left(\infty, \frac{u}{m n}\right)=O\left(\frac{u}{m n}, \infty\right)$ and so $G_{u, m n}$ is self-paired.

If $\frac{r}{s n} \rightarrow \frac{x}{y n}$ in $G_{u, m n}$, then Theorem 3.1 implies that $r y-s x= \pm m$, so $\frac{r}{s n} \approx \frac{x}{y n}$. Thus each connected component of $G_{u, m n}$ lies in a single block for $\approx$, of which there are $\psi(n)$, so we have:

Corollary 3.3. The graph $G_{u, m n}$ is a disjoint union of $\psi(n)$ subgraphs.

\section{Subgraph $£_{\mathrm{u}, \mathrm{n}}$}

In this section, we will investigate suborbital graph $G_{u, n}$ by taking $m=1$ in the graph $G_{u, m n}$. As we see, each $G_{u, n}$ is a disjoint union of $\psi(n)$ subgraphs, the vertices of each subgraph forming a single block with respect to the relation $\approx$. Since $\Gamma^{*}(n)$ acts transitively on $\hat{\mathbb{Q}}_{n}$, it permutes these blocks transitively, so the subgraphs are all isomorphic. Let $£_{u, n}$ be the subgraph of $G_{u, n}$ whose vertices form the block [ $\infty$ ], so that $G_{u, n}$ consists of $\psi(n)$ disjoint copies of $£_{u, n}$. The elements of $£_{u, n}$ has the form $\frac{a n+1}{b n}$ where $a, b \in \mathbb{Z}$. And also Farey graph is the same graph with $£_{1,1}$ graph.

Now let us give the conditions of being an edge in $£_{u, n}$ graph;

Theorem 3.3. Let $\frac{r}{s n}$ and $\frac{x}{y n}$ be in the block [ $\infty$. Then there is an edge $\frac{r}{s n} \rightarrow \frac{x}{y n}$ in $£_{u, n}$ if and only if either

(i) $x \equiv u r\left(\operatorname{modn} n^{2}\right)$ and $r y-s x=1$ or

(ii) $x \equiv-u r\left(\operatorname{modn} n^{2}\right)$ and $r y-s x=-1$.

Proof. It is clear from Theorem 3.1.

An automorphism of the graph $£_{u, n}$ is a permutation of $[\infty]$ which takes edges to edges. In view of this it can easily seen that $\Gamma(n)<A u t £_{u, n}$.

Theorem 3.4. $\Gamma(n)$ permutes the vertices and the edges of $£_{u, n}$ transitively.

Proof. Suppose that $u, v \in[\infty]$. As $\Gamma^{*}(n)$ acts on $\hat{\mathbb{Q}}_{n}$ transitively, $g(u)=v$ for some $g=\left(\begin{array}{cc}a & b n \\ c n & d\end{array}\right) \in \Gamma^{*}(n)$. Since $u \approx \infty$ and $\approx$ is $\Gamma^{*}(n)$-invariant equivalence relation, $g(u) \approx g(\infty)$; that is $v \approx g(\infty)$. Thus, as $v \approx g(\infty), g \in \Gamma(n)$.

Assume that $v, w \in[\infty] ; k_{1}, k_{2} \in[\infty]$ and $v \rightarrow w, k_{1} \rightarrow k_{2} \in £_{u, n}$. Then $(v, w),\left(k_{1}, k_{2}\right) \in O\left(\infty, \frac{u}{m n}\right)$. Therefore, for some $S, T \in \Gamma^{*}(n)$;

$$
S(\infty)=v, S\left(\frac{u}{m n}\right)=w \text { and } T(\infty)=k_{1}, T\left(\frac{u}{m n}\right)=k_{2} .
$$

Hence $S, T \in \Gamma(n)$ as $S(\infty), T(\infty) \in[\infty]$. We see that, furthermore $T S^{-1}(v)=k_{1}$ and $T S^{-1}(w)=k_{2}$; that is $T S^{-1} \in \Gamma(n)$. 
Corollary 3.4. No edges of $£_{u, n}$ cross in $\mathbb{H}$.

Proof. Without loss of generality, since the action on $\hat{\mathbb{Q}}_{n}$ is transitive, suppose that $\infty \rightarrow \frac{u}{n}, \frac{x_{1}}{n n_{1}} \rightarrow \frac{x_{2}}{n n_{2}}$ and $\frac{x_{1}}{n n_{1}}<\frac{u}{n}<\frac{x_{2}}{n n_{2}}$, where all letters are positive integer.

Since $\frac{x_{1}}{n n_{1}} \rightarrow \frac{x_{2}}{n n_{2}}$ and $\frac{x_{1}}{n n_{1}}<\frac{u}{n}<\frac{x_{2}}{n n_{2}}$ then $n n_{2} x_{1}-n n_{1} x_{2}=-1$ and $\frac{x_{1}}{n_{1}}<u<\frac{x_{2}}{n_{2}}$ respectively. Therefore $\frac{x_{1}}{n_{1}}-\frac{x_{2}}{n_{2}}<u-\frac{x_{2}}{n_{2}}<0$. Then $\frac{x_{1} n_{2}-x_{2} n_{1}}{n_{1}}<u n_{2}-x_{2}<0$. So $-\frac{1}{n n_{1}}<u n_{2}-x_{2}<0$ is obtained. This is impossible.

Definition 3.2. Let $£_{u, n}$ and $£_{v, k}$ be two suborbital graphs. If the map $\mu$ is a bijective function from the vertex set of $£_{u, n}$ to that of $£_{v, k}$ and sends the edges of $£_{u, n}$ to the edges of $£_{v, k}$ then $\mu$ is called a suborbital graph isomorphism and it will be denoted by $\mu: £_{u, n} \rightarrow £_{v, k}$.

Lemma 3.2. (i) There is a suborbital graph isomorphism $£_{u, n} \rightarrow £_{-u, n}$ given by $v \rightarrow-v$ for all vertices $v$ of $£_{u, n}$.

(ii) If $k \mid n$ then there is a suborbital graph isomorphism from $£_{u, n}$ to a subgraph of $£_{u, k}$, given by $\mu(v)=\frac{n v}{k}$ for all vertices $v$ of $£_{u, n}$.

Corollary 3.5. There is a suborbital graph isomorphism from $£_{u, n}$ to a subgraph of $£_{1,1}$, given by $\mu(v)=n v$ for all vertices $v$ of $£_{u, n}$ if and only if $k=1$.

\section{Main Calculations}

In this last section, we examine the connectedness of $£_{u, n}$.

Definition 4.1. A subgraph $K$ of $G_{u, n}$ is called connected if any pair of its vertices can be joined by a path in $K$.

Theorem 4.1. $£_{1,2}$ is connected.

Proof. We will show that each vertex $v=\frac{a}{2 b} b \geq 1,(a, 2 b)=1$ of $£_{1,2}$ is joined to $\infty$ by a path in $£_{1,2}$. Since the pattern is periodic with period 2, we can show by induction on $b$. If $b=1$, then $v=\frac{a}{2}$ can be joined with $\infty$. If $a=1$, it is clear that $\frac{1}{0} \rightarrow \frac{1}{2}$. If $a=3$, then $\frac{3}{2} \rightarrow \frac{1}{0}$ because $1 \equiv-3(\bmod 4)$ and $3 \cdot 0-2 \cdot 1=-2$. If $a=5$, then $\frac{1}{0} \rightarrow \frac{5}{2}$. The same holds for the rest periodically. So we can assume that $b \geq 2$.

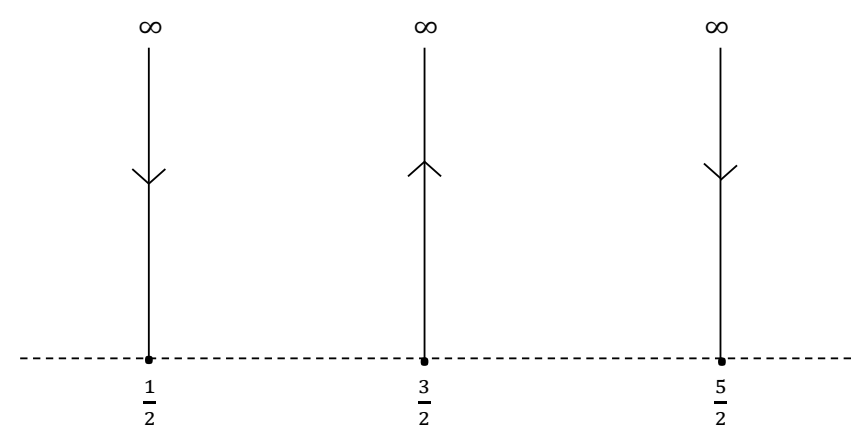

Figure 2. $£_{1,2}$ graph

To complete the proof, we show that $v$ is adjacent to a vertex $w=\frac{a}{2 b_{1}}$ with $b_{1}<b$. It means that, $w$ is connected by a path to $\infty$, and hence so is $v$. As $(a, b)=1$, there exist integers $c, d$ such that $a d-b c=1$. For some $k \in \mathbb{Z}$, replacing $c$ and $d$ by $c+k a$ and $d+k b$, without loss of generality we can suppose that $0<d<b$.

(i) If $c$ is odd, then $w=\frac{c}{2 d}$ can be joined with $\frac{a}{2 b}$. Indeed, $\frac{a}{2 b} \stackrel{>}{\longrightarrow} \frac{c}{2 d}$ gives that $a \cdot 2 d-c \cdot 2 b=2$ and $c \equiv a(\bmod 4)$. If $c \not \equiv a(\bmod 4)$, taking $c \equiv-a(\bmod 4)$ we obtain $\frac{a}{2 b} \longleftarrow \frac{c}{2 d}$ by $2 b c-2 a d=-2$. Hence, if $c$ is odd, $\frac{a}{2 b}$ is adjacent to $\frac{c}{2 d}$ in $£_{1,2}$.

(ii) If $c$ is even, then $a-c$ is odd. As $0<b-d<b$, we can take $w=\frac{a-c}{2(b-d)}$, adjacent to $\frac{a}{2 b}$.If $\frac{a}{2 b} \stackrel{<}{\longrightarrow} \frac{a-c}{2(b-d)}$ then $2(b c-a d)=-2$ and $2 a-c \equiv 0(\bmod 4)$. Here, if $2 a-c \not \equiv 0(\bmod 4)$ then this edge must be $\frac{a-c}{2(b-d)} \longrightarrow \frac{a}{2 b}$ and so we have that $c \equiv 0(\bmod 4)$ and $2(a d-b c)=2$.

Consequently, $£_{1,2}$ is connected. 
Corollary 4.1. $£_{3,2}$ is connected.

Proof. By the suborbital graph isomorphism $£_{1,2} \underset{v}{\longrightarrow} \underset{-v}{£_{-1,2}}=£_{3,2}, £_{3,2}$ is also connected.

Theorem 4.2. If $n \geq 3$, then $£_{u, n}$ is not connected.

Proof. Since $n \geq 3$ and $u \equiv 1(\bmod n)$ it has the form $u=k n+1$. Also $k+\frac{1}{2}$ and $k+1$ are not in [ $\infty$ ]. We will show that vertices of $£_{u, n}$ which between lines $\operatorname{Re} z=k+\frac{1}{2}$ and $\operatorname{Re} z=k+1$ can not adjacent with vertices which are outside the lines. Of course, there is at least some vertex of $£_{u, n}$ in this strip.Such as there is $k+\frac{n+1}{2 n}$ elements of $£_{u, n}$. Suppose there is an edge $\frac{m n+1}{s n} \rightarrow \frac{l n+1}{y n}$ for $\frac{m n+1}{s n}<\frac{2 k+1}{2}<\frac{l n+1}{y n}$. Then we have $\frac{m n+1}{s}<\frac{n(2 k+1)}{2}<\frac{\ln +1}{y}$.

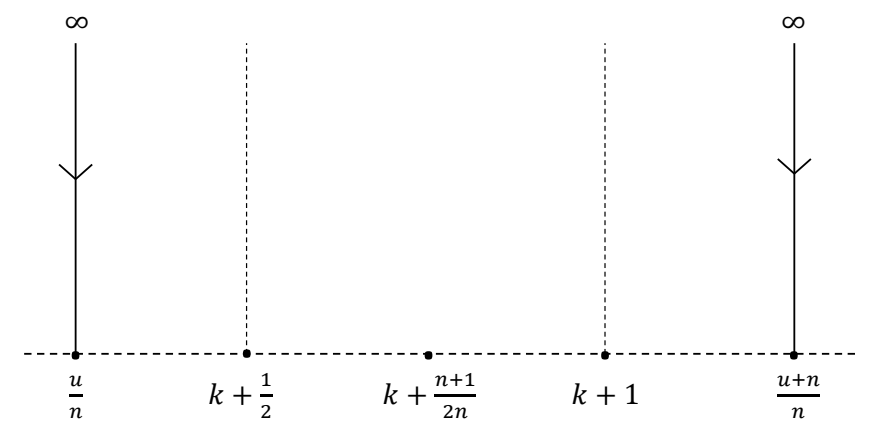

Figure 3. $£_{u, n}$ graph

(i) If $n$ is even then $\frac{n(2 k+1)}{2} \in \mathbb{Z}$. Thus the edges $\frac{m n+1}{s} \rightarrow \frac{l n+1}{y}$ with $\infty \rightarrow \frac{n(2 k+1)}{2}$ cross in $£_{1,1}$. This is a contradiction. So $n$ is not even.

(ii) If $n$ is odd then there exists $t \in \mathbb{Z}$ such that $\frac{n(2 k+1)}{2}=\frac{(2 t+1)(2 k+1)}{2}=2 t k+t+k+\frac{1}{2}$ is obtained. Therefore $s=y=1$. Also $m n+1=2 t k+t+k$ and $2 t k+t+k+1=l n+1$ are held. From $2 t k+t+k=l n, n \mid 2 t k+t+k=m n+1$. So $n \mid 1$ is obtained.This is impossible because of $n \geq 3$. It means that no vertices of $£_{u, n}$ between $\operatorname{Re} z=k+\frac{1}{2}$ and $\operatorname{Re} z=k+1$ can not connect with vertices which are outside the lines. Consequently, $£_{u, n}$ is not connected.

Theorem 4.3. $£_{u, n}$ is connected if and only if $n \leq 2$.

Proof. If $n=1,2$ then the result follows from [1], otherwise it follows from Theorem 4.2.

Theorem 4.4. If $n>1$, then there is no triangle in $£_{u, n}$.

Proof. Assume that $£_{u, n}$ contains a directed triangle. Since $\Gamma^{*}(n)$ acts transitively on $\hat{\mathbb{Q}}_{n}$, the form of directed triangle can be taken as $\infty \rightarrow \frac{u}{n} \rightarrow \frac{x}{n} \rightarrow \infty$ for some integer $x$. By using Theorem 3.3, as $\frac{u}{n} \rightarrow \frac{x}{n}$, then $u-x= \pm 1$ and $x=u+1$ or $x=u-1$.

If $x=u+1$ then $\infty \rightarrow \frac{u}{n} \rightarrow \frac{u+1}{n} \rightarrow \infty$. From $\frac{u+1}{n} \rightarrow \infty, 1 \equiv-u(u+1)\left(\bmod n^{2}\right)$ is achieved.That is, $u^{2}-u+1 \equiv 0\left(\bmod n^{2}\right)$. Since $\frac{u}{n} \in[\infty], 1 \equiv u(\bmod n)$. Thus $1 \equiv 0(\bmod n)$ is obtained. Hence $n=1$ which is a contradiction. Similarly, $\infty \rightarrow \frac{u}{n} \rightarrow \frac{u-1}{n} \rightarrow \infty$, the second case can be shown.As a result there is not directed triangle in $£_{u, n}$.

In addition, $\infty \rightarrow \frac{u}{n} \rightarrow \frac{u+1}{n} \leftarrow \infty$ or $\infty \rightarrow \frac{u}{n} \rightarrow \frac{u-1}{n} \leftarrow \infty$ can not be hold. Indeed, if $\frac{u+1}{n} \leftarrow \infty$ then $u+1 \equiv u\left(\bmod n^{2}\right)$ and so $1 \equiv 0\left(\bmod n^{2}\right)$ is obtained. This is impossible. Likewise the case $\frac{u-1}{n} \leftarrow \infty$ is also a contradiction. Last of all, for $n>1$ there is no triangle in $£_{u, n}$.

Theorem 4.5. $£_{u, n}$ contains a triangle if and only if $n=1$.

Proof. Let $\kappa$ be a triangle in $£_{u, n}$. We may suppose that $\kappa$ has the form $\infty \rightarrow v_{1} \rightarrow v_{2} \rightarrow \infty$ or $\infty \rightarrow v_{1} \leftarrow v_{2} \rightarrow \infty$. Let us make calculations only for the first triangle. We easily seen that $v_{1}=\frac{x}{n}$ and $v_{2}=\frac{y}{n}$ for some $x, y \in \mathbb{Z}$. By using Theorem 3.3, if $\frac{x}{n} \longleftrightarrow \frac{y}{n}$ then $x n-y n=-1$. Since $\frac{x}{n}, \frac{y}{n} \in[\infty], x \equiv y(\bmod n)$. So $x-y \equiv 0(\bmod n)$ is obtained. Therefore $n=1$. If $\frac{x}{n} \gg \frac{y}{n}$ then $x n-y n=1$. And so, again, $n=1$. Conversely if $n=1$ then we have the triangle $\frac{1}{0} \rightarrow \frac{1}{1} \rightarrow \frac{2}{1} \rightarrow \frac{1}{0}$.

Lemma 4.1. If $£_{u, n}$ contains a triangle then, $\Gamma(n)$ contains an elliptic element of order 3. 
Proof. If $£_{u, n}$ contains a triangle, then by Theorem $4.5 n=1$ is achieved. So $\Gamma(1)=\Gamma^{*}(1)=\Gamma$ and $\phi:=\left(\begin{array}{cc}1 & -3 \\ 1 & -2\end{array}\right) \in$ $\Gamma(1)$ is an elliptic element of order 3 . That is, $\phi^{3}=I$. And also, it is clear that

$$
\phi(\infty)=1, \quad \phi(1)=2, \quad \phi(2)=\infty .
$$

The transformation $\phi$ establish a connection between circuits in the graph and elliptic elements in the graph $\Gamma(n)$.

In general, the converse of Lemma 4.1 may not true.

Remark 4.1. One can show the similar conclusion in the Theorem 3.3 for the block [0]. Without loss of generality, since the equation

$$
\left(\begin{array}{cc}
a & b n \\
c n & d
\end{array}\right)\left(\begin{array}{ll}
0 & n \\
1 & u
\end{array}\right)=\left(\begin{array}{cc}
s n & y n \\
r & x
\end{array}\right)
$$

holds for $T=\left(\begin{array}{cc}a & b n \\ c n & d\end{array}\right) \in \Gamma(n), x \equiv \pm u r\left(\bmod n^{2}\right)$ and $r y-s x= \pm 1$ are obtained.

Example 4.1. Let $u=1$. Then the triangle circuits in $£_{1,1}$ and $\kappa_{1,1}$ denote the subgraphs in $G_{1,1}$ whose vertices form the block $[\infty]$ and $[0]$ respectively.
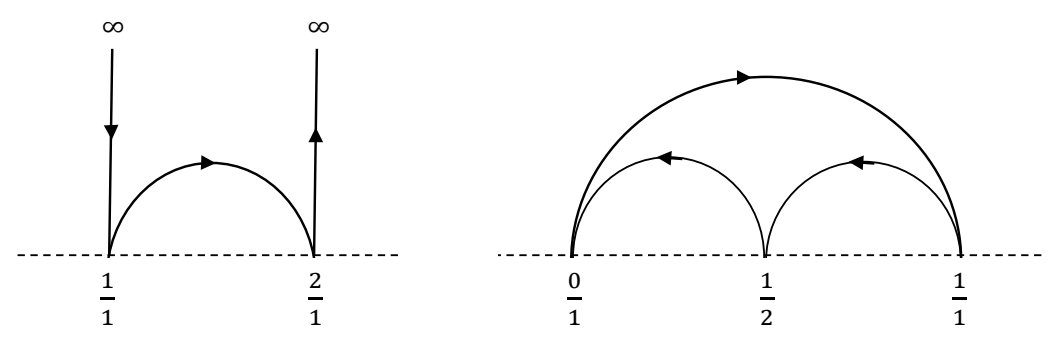

Figure 4. Directed Triangles in $[\infty]$ and $[0]$

\section{References}

[1] Jones, G. A., Singerman, D. and Wicks, K.,The modular group and generalized Farey graphs. London Mathematical Society Lecture Note Series. 160 (1991), 316-338.

[2] Akbaş, M.,On suborbital graphs for the modular group. Bulletin London Mathematical Society. 33 (2001), 647-652.

[3] Bigg, N.L. and White, A.T., Permutation groups and combinatorial structures. London Mathematical Society Lecture Note Series 33. Cambridge University Press, Cambridge, 1979.

[4] Sims, C.C., Graphs and finite permutation groups. Mathematische Zeitschrift. 95 (1967),76-86.

[5] Jones, G. A. and Singerman, D., Complex functions: an algebraic and geometric viewpoint. Cambridge University Press, Cambridge, 1987.

[6] Schoeneberg, B., Elliptic modular functions. Springer, Berlin, 1974.

[7] Newman, M., Classification of normal subgroups of the modular group.American journal of Mathematics. 126 (1967), 267-277.

[8] Tsuzuku, T., Finite Groups and Finite Geometries. Cambridge University Press, Cambridge, 1982.

[9] Dixon, J.D. and Mortimer, B., Permutation groups. Springer-Verlag, New York, 1996.

[10] Hardy, G.H. and Wright, E.M., An introduction to the theory of numbers. Oxford University Press, Oxford, 1979. 
[11] Rankin, R.A., Modular forms and functions. Cambridge University Press, Cambridge, 2008.

[12] Kesicioğlu, Y., Akbaş, M. and Beşenk, M., Connectedness of a suborbital graph for congruence subgroups. Journal of Inequalities and Applications. 1 (2013), 117-124.

[13] Güler, B.Ö., Beşenk, M., Değer, A.H. and Kader, S., Elliptic elements and circuits in suborbital graphs. Hacettepe Journal of Mathematics and Statistics. 40 (2011),no. 2, 203-210.

[14] Beşenk, M., Güler, B.Ö., Değer, A.H. and Kesicioğlu, Y., Circuit lengths of graphs for the Picard gorup. Journal of Inequalities and Applications. 1 (2013), 106-114.

\section{Affiliations}

MuRAT BEŞENK

ADDRESS: Karadeniz Technical University, Faculty of science, Department of Mathematics, 61080 Trabzon-TURKEY.

E-MAIL: mbesenk@ktu.edu.tr 